\title{
BOURGIN-YANG-TYPE THEOREM FOR $a$-COMPACT PERTURBATIONS OF CLOSED OPERATORS. PART I. THE CASE OF INDEX THEORIES WITH DIMENSION PROPERTY
}

\author{
SERGEY A. ANTONYAN, ZALMAN I. BALANOV, AND BORIS D. GEL’MAN
}

Received 26 June 2005; Accepted 1 July 2005

A variant of the Bourgin-Yang theorem for $a$-compact perturbations of a closed linear operator (in general, unbounded and having an infinite-dimensional kernel) is proved. An application to integrodifferential equations is discussed.

Copyright (c) 2006 Sergey A. Antonyan et al. This is an open access article distributed under the Creative Commons Attribution License, which permits unrestricted use, distribution, and reproduction in any medium, provided the original work is properly cited.

\section{Introduction}

1.1. Goal. Among several different, but equivalent, formulations of the famous BorsukUlam theorem, the following one is of our interest: if $f: S^{n} \rightarrow \mathbb{R}^{n}$ is a continuous odd map, then there exists an $x \in S^{n}$ such that $f(x)=f(-x)=0$ (see [17] for other formulations, generalizations, and applications, and $[11,13]$ for a connection with the corresponding Brouwer degree results).

Under the "stronger" assumption that $f: S^{n} \rightarrow \mathbb{R}^{m}$, where $m<n$, one can expect that there are bigger coincidence sets. The results which measure the size of the set $A:=\{x \in$ $\left.S^{n} \mid f(-x)=f(x)\right\}$ in topological terms, like dimension, (co)homology, genus (or other index theory), are usually called "Bourgin-Yang theorems." The simplest result in this direction (cf. $[5,19]$ ) can be formulated as follows: (i) $\operatorname{dim} A(f) \geq n-m$ (covering or cohomological dimension) and (ii) $g(A(f)) \geq n-m+1$, where $g(\cdot)$ stands for the genus with respect to the antipodal action (see Example 2.4). We refer to [17] for extensions of this result to more complicated (finite-dimensional) $G$-spaces, where $G$ is a compact Lie group, as well as to index theories different from genus.

Holm and Spanier were the first to extend the Bourgin-Yang theorem to infinite dimensions (see [10], where the solution set to the equation $a(x)=f(x)$ was studied in the case $a$ is a proper $C^{\infty}$-smooth Fredholm operator and $f$ is a compact map; both equivariant with respect to a free involution). It should be pointed out that the assumptions on $a$ required in [10] allow a clear finite-dimensional reduction (the kernels and images in question are complementable). At the same time, the methods developed in [10] cannot be 
applied to treat the case when $F$ is not Fredholm. The first step in this direction was done in recent papers $[8,9]$, where the author studied the situation when $a$ is a continuous (resp., linear closed) linear operator without any restrictions with respect to $\operatorname{dim} \operatorname{ker}(a)$ (in fact, in these papers only, the "dimension part" of the Bourgin-Yang theorem was proved in the presence of the antipodal symmetry). The main new ingredient in $[8,9]$ allowing the author to go around the "complementability problem" is the application of the Michael selection theorem respecting the antipodal symmetry to the multivalued map $a^{-1}$. Observe, however, that the corresponding "equivariant selection theorem" was proved in [7] for free actions of a finite group-by no means to be extended to nonfree actions of compact Lie groups.

The main goal of our paper is to extend the results from [8-10] in several directions:

(i) $a$ is an arbitrary closed linear map (in general, unbounded, and having an infinite-dimensional kernel) equivariant with respect to arbitrary compact Lie group representations;

(ii) $f$ is a so-called $a$-compact $G$-equivariant map (see Definition 4.1);

(iii) the coincidence set is estimated in terms of an arbitrary index theory with the so-called "dimension property" (cf. [4, 17], [14, Chapter 5]).

To this end, based on the results from [1], we establish a general equivariant version of the Michael selection theorem (without any restrictions with respect to $G$-actions) which, in our opinion, is interesting in its own. This result allows us to construct for $a$ an equivariant section taking bounded sets to the bounded ones (see Lemma 3.6). Using this lemma, we reduce the coincidence problem to the fixed point problem.

1.2. Overview. After Section 1, the paper is organized as follows. In Section 2, we briefly discuss "index theories." Section 3 is devoted to the proof of the equivariant Michael selection theorem and Lemma 3.6. After the reduction to the fixed point problem (see Section 4), we prove the main result (Theorem 4.3) in Section 5. In the last section, we give an application of the main result to integrodifferential equations. For the equivariant jargon, frequently used in this paper, we refer to [6].

\section{Index theories}

Convention and notations. Hereafter, $G$ stands for a compact Lie group.

Without loss of generality, we will assume all Banach $G$-representations to be isometric.

Given a Banach $G$-representation $E$,

(i) $S_{R}$ stands for the sphere in $E$ of radius $R$ centered at the origin;

(ii) $E^{G}=\{x \in E \mid g x=x$, for all $g \in G\}$-the fixed point set.

Let us recall the standard construction of the join.

Definition 2.1. Let $X_{1}, \ldots, X_{n}$ be topological spaces and $\Delta^{n-1}=\left\{\left(t_{1}, \ldots, t_{n}\right) \in \mathbb{R}^{n} \mid 0 \leq t_{i} \leq\right.$ $\left.1, \sum_{i=1}^{n} t_{i}=1\right\}$ - the $(n-1)$-dimensional standard simplex. The join $X_{1} * \cdots * X_{n}$ is the quotient space of the product $X_{1} \times \cdots \times X_{n} \times \Delta^{n-1}$ under the following equivalence relation: $\left(x_{1}, \ldots, x_{n}, t_{1}, \ldots, t_{n}\right) \sim\left(x_{1}^{\prime}, \ldots, x_{n}^{\prime}, t_{1}^{\prime}, \ldots, t_{n}^{\prime}\right)$ if and only if $t_{i}=t_{i}^{\prime}(i=1, \ldots, n)$ and $x_{i}=x_{i}^{\prime}$ whenever $t_{i}=t_{i}^{\prime}>0$. 
It is convenient to denote a point of the join $X_{1} * \cdots * X_{n}$ in the form of a formal convex combination: $\sum_{i=1}^{n} t_{i} x_{i}$.

If $X_{1}=\cdots=X_{n}=X$, then write $J_{n} X$ for $X_{1} * \cdots * X_{n}$.

If $X_{1}, \ldots, X_{n}$ are $G$-spaces, then so is $X_{1} * \cdots * X_{n}$ via $g \cdot \sum_{i=1}^{n} t_{i} x_{i}:=\sum_{i=1}^{n} t_{i} g x_{i}$, $g \in G$.

Example 2.2. Obviously, $J_{n} S^{0}=S^{n-1}, J_{n} S^{1}=S^{2 n-1}$, and $J_{n} S^{3}=S^{4 n-1}$. Also, if we consider $S^{0}$ (resp., $S^{1}$ and $S^{3}$ ) as free $\mathbb{Z}_{2}$ - (resp., $S^{1}$ - and $S U(2)$-spaces), then the action of $\mathbb{Z}_{2}$ on $J_{n} S^{0}$ (resp., $S^{1}$ on $J_{n} S^{1}$ and $S U(2)$ on $J_{n} S^{3}$ ) corresponds to the antipodal action (resp.,

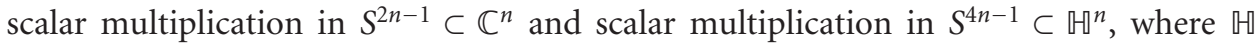
stands for the quaternions).

Following [4], [14, Chapter 5], [17], we give the following definition.

Definition 2.3. A function "ind" that assigns to every $G$-space $A$ a number $\operatorname{ind}(A) \in \mathbb{N} \cup$ $\{0\}$ or $\{\infty\}$ is called an index theory if it satisfies the following properties.

(i) ind $(A)=0$ if and only if $A=\varnothing$.

(ii) Subadditivity. If a $G$-space $A$ is the union of two of its closed invariant subsets $A_{1}$ and $A_{2}$, then ind $(A) \leq \operatorname{ind}\left(A_{1}\right)+\operatorname{ind}\left(A_{2}\right)$.

(iii) Continuity. If $A$ is a closed invariant subset of a $G$-space $X$, then there exists a closed invariant neighborhood $U$ of $A$ in $X$ such that ind $(A)=\operatorname{ind}(U)$.

(iv) Monotonicity. If $A_{1}$ and $A_{2}$ are two $G$-spaces and there exists an equivariant map $\varphi: A_{1} \rightarrow A_{2}$, then ind $\left(A_{1}\right) \leq \operatorname{ind}\left(A_{2}\right)$.

In particular, (a) if $A_{1} \subset A_{2}$, then ind $\left(A_{1}\right) \leq \operatorname{ind}\left(A_{2}\right)$, and (b) if $\varphi: A_{1} \rightarrow A_{2}$ is an equivariant homeomorphism, then ind $\left(A_{1}\right)=\operatorname{ind}\left(A_{2}\right)$.

Example 2.4 (genus). For a $G$-space $A$ set $g(A)=k$ if there exist closed subgroups $H_{1}, \ldots$, $H_{k}$ of $G, H_{i} \neq G, i=1, \ldots, k$, and a $G$-equivariant map $A \rightarrow G / H_{1} * \cdots * G / H_{k}$, where $k$ is minimal with this property ( $G$ acts on $G / H_{i}$ by left translations). If such $k$ does not exist, put $g(A):=\infty$. Also, $g(\varnothing)=0$.

It is easy to check (see [3]) that the function $g$ satisfies all the properties required for an index theory.

In fact, there is a "myriad" of nonequivalent index theories (mostly, cohomological (see [3] and references therein)).

In this paper, we are dealing with index theories satisfying an additional property (cf. [4], [14, Chapter 5], [17]). Namely, we have the following definition.

Definition 2.5 (dimension property). An index theory ind is said to satisfy the dimension property if there exists $d \in \mathbb{N}$ such that for any Banach $G$-representation $E$, one has ind $\left(E^{k d} \cap S_{1}\right)=k$ for all invariant $k d$-dimensional subspaces $E^{k d}$ of $E$ satisfying $E^{k d} \cap$ $E^{G}=\{0\}$.

As an immediate consequence of the dimension property, one has (cf. [4]) that ind $(A)$ $<\infty$ for any compact invariant subset $A \subset E$ of a Banach $G$-representation $E$ with $A \cap$ $E^{G}=\varnothing$. Although, in general, the genus does not satisfy the dimension property, there are some important (from the application point of view) classes of groups for which it does (see the examples following below). 
Example 2.6. (i) If $G=\mathbb{Z}_{p} \times \cdots \times \mathbb{Z}_{p}$ ( $p$ is prime), then the genus satisfies the dimension property with $d=1$ (cf. [3]).

(ii) If $G=S^{1} \times \cdots \times S^{1}$, then the genus satisfies the dimension property with $d=2$ (cf. [3]).

Remark 2.7. Restricting the genus to free $G$-spaces, one can define a "restricted index theory" satisfying the dimension property with $d=1+\operatorname{dim} G$ (cf. [3]). Recall that if $G$ acts freely on a finite-dimensional sphere, then $G$ is either finite, or $S^{1}$, or $S^{3}$, or the normalizer of $S^{1}$ in $S^{3}$ (cf. [6, Chapter 4, Theorem 6.2]). All finite groups admitting a free action on a finite-dimensional sphere are described in [18].

\section{Equivariant selection theorem}

We begin this section with recalling the Michael selection theorem. To this end, we need several definitions.

Definition 3.1. (i) Let $X$ and $Y$ be topological spaces. It will be said that $F$ is a multivalued map from $X$ to $Y$ if $F$ associates with each point $x \in X$ a nonempty subset $F(x)$ of $Y$. If, in addition, $X$ and $Y$ are $G$-spaces, then $F$ is called a multivalued $G$ map or a multivalued equivariant map, if $F(g x)=g F(x)$ for all $g \in G$ and $x \in X$, where $g F(x)=\{g y \mid y \in F(x)\}$.

(ii) A multivalued map $F$ from $X$ to $Y$ is called lower semicontinuous (l.s.c.) if for any open subset $U \subset Y$, the set

$$
F^{-1}(U)=\{x \in X \mid F(x) \cap U \neq \varnothing\}
$$

is open in $X$.

Definition 3.2. (i) A continuous (single-valued) map $f: X \rightarrow Y$ is called a selection for a multivalued map $F$ from $X$ to $Y$ if $f(x) \in F(x)$ for all $x \in X$.

(ii) Assume $X$ and $Y$ are $G$-spaces and $F$ is a multivalued $G$-map. A selection $f$ of $F$ is called a $G$-selection if, in addition, $f$ is a $G$-map.

The following fact is well known as the Michael selection theorem.

Theorem 3.3 (see [15]). Let $X$ be a paracompact space, $Y$ a Banach space, and $F$ an l.s.c. multivalued map from $X$ to $Y$ such that $F(x)$ is a nonempty, closed, convex set for all $x \in X$. Then $F$ admits a selection.

Below, we formulate and prove an equivariant version of the Michael selection theorem.

Theorem 3.4. Let $X$ be a paracompact G-space, $Y$ a Banach $G$-representation, and $F$ a multivalued l.s.c. G-map from $X$ to $Y$ such that for all $x \in X, F(x)$ is a closed, convex set. Then $F$ admits a G-selection.

Proof. According to the Michael selection theorem (Theorem 3.3), there exists a continuous selection $f: X \rightarrow Y$ of $F$. Let $d g$ be the normalized Haar measure on G. Define a new 
single-valued map $\varphi: X \rightarrow Y$ by

$$
\varphi(x)=\int_{G} g^{-1} f(g x) d g, \quad x \in X,
$$

(the symbol on the right-hand side denotes the vector-valued integral with respect to the Haar measure).

We claim that $\varphi$ is the desired $G$-selection of $F$. Indeed, since $f(g x) \in F(g x)=g F(x)$, we see that $g^{-1} f(g x) \in g^{-1}(g F(x))=F(x)$ for all $g \in G$. Since $F(x)$ is a closed convex set, we infer that the closed convex hull $\overline{\operatorname{conv}\left(A_{f}\right)}$ of the set $A_{f}:=\left\{g^{-1} f(g x) \mid g \in G\right\}$ is contained in $F(x)$. But the above integral belongs to $\overline{\operatorname{conv}\left(A_{f}\right)}$ (see [16, Part 1, Theorem 3.27]). This yields that $\varphi(x) \in F(x)$.

Continuity and equivariance of the map $\varphi: X \rightarrow Y$ can be easily derived from the corresponding properties of the integral presented in the following lemma

Lemma 3.5 (see [1]). Assume that $V$ is a complete (in the sense of the natural uniformity induced from $Z$ ) convex invariant subset of a locally convex topological vector space $Z$ on which a compact group $G$ acts linearly. Let $C(G, V)$ denote the set of all continuous maps $f: G \rightarrow V$ endowed with the compact-open topology. Then the vector-valued Haar integral $\int: C(G, V) \rightarrow V$ is a well-defined continuous map satisfying the following properties:

(a) $\int_{h} f=\int f=\int f_{h}$ for any $f \in C(G, V)$ and any $h \in G$, where ${ }_{h} f(g)=f(h g)$ and $f_{h}(g)=f(g h)$ for all $g \in G$;

(b) $\int g * f=g \int f$ for any $f \in C(G, V)$ and any $g \in G$, where the action $g * f$ of $G$ on $C(G, V)$ is defined by $(g * f)(x)=g f(x), x \in G$;

(c) $\int f=v_{0}$, if $f(G)=\left\{v_{0}\right\}$ for a point $v_{0} \in V$.

Also, assuming in addition that $G$ is finite or $Z$ is finite-dimensional, one can remove the completeness requirement on $V$.

Next, we will apply Theorem 3.4 to prove the existence of a special $G$-selection of a linear $G$-equivariant closed map of Banach $G$-representations.

Let $E_{1}$ and $E_{2}$ be Banach spaces, $a: D(a) \subset E_{1} \rightarrow E_{2}$ a linear closed surjective map. Take the natural projection $p: E_{1} \rightarrow E_{1} / \operatorname{Ker}(a):=\overline{E_{1}}$ and consider the (invertible) map $a_{1}: D\left(a_{1}\right) \subset \overline{E_{1}} \rightarrow E_{2}$, where $D\left(a_{1}\right):=p(D(a))$ and $a_{1}([x]):=a(x)$. Put (see, e.g., $\left.[8,9]\right)$

$$
\beta(a):=\sup _{y \in E_{2} \backslash\{0\}} \frac{\left\|a_{1}^{-1}(y)\right\|}{\|y\|}=\sup _{y \in E_{2} \backslash\{0\}} \frac{\inf \left\{\|x\| \mid x \in E_{1}, a(x)=y\right\}}{\|y\|} .
$$

Lemma 3.6. Let $E_{1}$ and $E_{2}$ be Banach isometric G-representations, $a: D(a) \subset E_{1} \rightarrow E_{2} a$ $G$-equivariant linear closed surjective map, and $k>\beta(a)(c f .(3.3))$. Then there exists $a G$ equivariant continuous map $q: E_{2} \rightarrow E_{1}$ satisfying the following conditions:

(i) $a(q(y))=y$ for all $y \in E_{2}$;

(ii) $q(y) \leq k\|y\|$ for all $y \in E_{2}$.

Proof. Denote by $a^{-1}$ a multivalued map from $E_{2}$ to $E_{1}$ "inverse" to $a$, that is, $a^{-1}$ assigns to each $y \in E_{2}$ its full inverse image under $a$. Obviously, $a^{-1}$ is a multivalued $G$-map with nonempty closed convex values. Moreover (cf. [2, Chapter 3], [8, 9]), $a^{-1}$ is 1.s.c. (even Lipschitzian with the Lipschitz constant $\beta(a))$. 
Consider together with $a^{-1}$ another multivalued map $\Phi$ from $E_{2}$ to $E_{1}$ defined by $\Phi(y):=B_{r(y)}[0]$, where $B_{r(y)}[0]$ is the closed ball of radius $r(y)=\beta(a)\|y\|+1$ centered at the origin of $E_{1}$. Obviously, $\Phi$ is also $G$-equivariant. Put $F(y):=a^{-1}(y) \cap \Phi(y)$. Still $F$ is a $G$-equivariant l.s.c map with nonempty closed convex values.

By Theorem 3.4, there exists a G-equivariant selection $q: E_{2} \rightarrow E_{1}$ of $F$. By construction, $q$ is as required.

Remark 3.7. Lemma 3.6 is quite obvious in the case $\operatorname{dim} \operatorname{ker}(a)<\infty$. Indeed, one has a direct sum decomposition $E_{1}=V \oplus \operatorname{ker}(a)$ and $V$ is isomorphic to $E_{2}$ as a $G$-representation. However, in general, $\operatorname{ker}(a)$ is not complementable and, therefore, one can think of $q$ as a nonlinear equivariant replacement for the corresponding $G$-isomorphism (the use of $G$-selections in this case seems to be unavoidable).

\section{Main result: formulation and reduction to a fixed point problem}

To formulate the main result of this paper (see Theorem 4.3), we need some preliminaries.

Definition 4.1. Let $E_{1}, E_{2}$ be Banach spaces, $a: D(a) \subset E_{1} \rightarrow E_{2}$ a closed surjective linear map. A continuous map $g: X \subset E_{1} \rightarrow E_{2}$ is said to be a-compact if the set $g\left(B \cap a^{-1}(A)\right)$ is compact for any bounded sets $A \subset E_{2}$ and $B \subset X$ (the empty set is compact by definition).

To give a simple criterion for the $a$-compactness of $g$, recall that the graph norm makes $D(a)$ a Banach space, denoted by $\widetilde{E}$. Clearly, the embedding $j: \widetilde{E} \rightarrow E_{1}$ is continuous. Put $\tilde{X}:=j^{-1}(X)$ and consider the map $\tilde{g}: \tilde{X} \rightarrow E_{2}$ defined by $\tilde{g}(x)=g(j(x))$.

Proposition 4.2. Under the above notations, $g$ is a-compact if and only if $\tilde{g}$ is compact.

As the proof of this proposition is straightforward, we omit it.

Here is our main result.

Theorem 4.3. Take an index theory ind satisfying the dimension property with some natural number d (cf. Definitions 2.3 and 2.5). Let $E_{1}, E_{2}$ be Banach G-representations and $E_{2}^{G}=\{0\}$. Let, further, $a: D(a) \subset E_{1} \rightarrow E_{2}$ be a closed surjective G-equivariant linear map such that $E_{1}^{G}$ is a proper finite-dimensional subspace of $\operatorname{ker}(a)$, and denote by $p$ the codimension of $E_{1}^{G}$ in $\operatorname{ker}(a)$ (the case $p=\infty$ is not excluded). Let $f: D(f) \subset S_{R} \rightarrow E_{2}$ satisfy the following conditions:

(i) $D(f)=D(a) \cap S_{R}$;

(ii) $f$ is G-equivariant;

(iii) $f$ is a-compact.

Denote by $N(a, f)$ the solution set to the equation

$$
a(x)=f(x)
$$

Then,

$$
\text { ind }(N(a, f)) \geq \frac{p}{d} \text {. }
$$


The proof of Theorem 4.3 will be given in the next section. Here, by means of Lemma 3.6, we will reduce the study of (4.1) to a G-equivariant fixed point problem with a compact operator.

By assumption, $E_{1}^{G}$ is finite-dimensional, hence we have a direct sum $G$-decomposition $E_{1}=E_{1}^{G} \oplus \widetilde{E}_{1}$. Put $\tilde{a}:=\left.a\right|_{\widetilde{E}_{1} \cap D(a)}$-the restriction. Since, by assumption, $E_{1}^{G} \subset \operatorname{ker}(a)$, we still have that $\tilde{a}$ is a closed $G$-equivariant surjective map. Let $q: E_{2} \rightarrow \widetilde{E}_{1}$ be the map provided by Lemma 3.6 (applied to $\tilde{a}$ ).

Next, define the map $g: D(a) \cap \widetilde{E}_{1} \rightarrow E_{2}$ by

$$
g(x)= \begin{cases}\frac{\|x\|}{R} f\left(\frac{R x}{\|x\|}\right), & x \neq 0, \\ 0, & x=0 .\end{cases}
$$

Further, take a direct sum $G$-decomposition $\operatorname{ker}(a)=E_{1}^{G} \oplus U(\operatorname{dim} U=p)$, consider the Banach $G$-representation $E:=E_{2} \oplus U$ equipped with diagonal $G$-action and the norm $\|(y, u)\|=\|y\|+\|u\|$, and define the map $\alpha: E \rightarrow E_{2}$ by $\alpha(y, u):=g(q(y)+u)$. Since $q$ and $g$ are equivariant, so is $\alpha$. Let us show that $\alpha$ is a compact map.

Take a bounded set $A \subset E$. Without loss of generality, one can assume that $A=A_{1} \times U_{1}$ with $A_{1} \subset E_{2}$ and $U_{1} \subset U$. By Lemma 3.6(ii), the set $A_{2}:=\{q(y)+u \mid(y, u) \in A\}$ is also bounded. Obviously, $A_{2} \subset a^{-1}\left(A_{1}\right)$. By the $a$-compactness of $g$, one concludes that the set $g\left(A_{2}\right)=\alpha(A)$ is compact.

Finally, take the unit sphere $S \subset E$ and consider the equation

$$
\alpha(y, u)=y \quad(y, u) \in S .
$$

LEMMA 4.4. Let $N(\alpha)$ be the solution set to (4.4), and define the map $\gamma: N(\alpha) \subset S \subset E \rightarrow$ $S_{R} \subset E_{1}$ by $\gamma(y, u):=R((q(y)+u) /\|q(y)+u\|)$. Then

(i) $\gamma$ is an equivariant homeomorphism onto its image;

(ii) $\gamma(N(\alpha)) \subset N(a, f)$.

Statement (i) follows immediately from Lemma 3.6(i). To show statement (ii), take $\left(y_{0}, u_{0}\right) \in S$ being a solution to (4.4). Obviously, $z_{0}:=q\left(y_{0}\right)+u_{0} \neq 0$. By direct computation,

$$
f\left(\frac{R z_{0}}{\left\|z_{0}\right\|}\right)=\frac{R}{\left\|z_{0}\right\|} y_{0} .
$$

On the other hand, using the linearity of $a$, one obtains

$$
a\left(z_{0}\right)=y_{0}
$$

Combining (4.5) and (4.6) yields $x_{0}:=R z_{0} /\left\|z_{0}\right\| \in N(a, f)$.

\section{Proof of the main result (Theorem 4.3)}

Throughout this section, we keep the same notations as in the previous section (in particular, $\operatorname{ker}(a)=E_{1}^{G} \oplus U$ and $\left.E:=E_{2} \oplus U\right)$. The proof of Theorem 4.3 splits into three steps. 
Step 1 (finite-dimensional case). Under the assumptions of Theorem 4.3, suppose that $\operatorname{dim} E<\infty$ and consider the equivariant map $\Phi: S \subset E \rightarrow E_{2} \subset E$ defined by $\Phi(y, u):=$ $\alpha(y, u)-y$. Then $N(\alpha)=\Phi^{-1}(0)$. By the continuity property of ind, there exists a closed neighborhood $U$ of $N(\alpha)$ such that

$$
\text { ind }(N(\alpha))=\operatorname{ind}(\mathcal{U}) \text {. }
$$

By the subadditivity property, one has

$$
\operatorname{ind}(S) \leq \operatorname{ind}(\widetilde{U})+\operatorname{ind}(\overline{S \backslash \bar{U}})
$$

Combining (5.1) and (5.2) yields

$$
\text { ind }(N(\alpha)) \geq \operatorname{ind}(S)-\operatorname{ind}(\overline{S \backslash \mathcal{U}}) \text {. }
$$

Observe that the equivariant map $\Phi$ takes $S \backslash \mathcal{U}$ to $E_{2} \backslash\{0\}$. Therefore, by the monotonicity property,

$$
\operatorname{ind}(\overline{S \backslash \bar{U}}) \leq \text { ind }\left(E_{2} \backslash\{0\}\right)
$$

Further, $S \cap E_{2}$ is a $G$-retract of $E_{2} \backslash\{0\}$, therefore, it follows from (5.4) and monotonicity property that

$$
\operatorname{ind}(\overline{S \backslash \mathcal{U}}) \leq \operatorname{ind}\left(S \cap E_{2}\right) \text {. }
$$

Combining (5.3) and (5.5) yields

$$
\text { ind }(N(\alpha)) \geq \operatorname{ind}(S)-\text { ind }\left(S \cap E_{2}\right) \text {. }
$$

Finally, using (5.6) and the dimension property of ind, one obtains

$$
\text { ind }(N(\alpha)) \geq \frac{\operatorname{dim} E}{d}-\frac{\operatorname{dim} E_{2}}{d}
$$

and the result follows in the considered case. 
Step 2 (finite-dimensional kernel). Under the assumptions of Theorem 4.3, suppose that $\operatorname{dim} U<\infty$ and reduce this situation to the previous step.

Put $X:=\overline{\operatorname{conv}(\alpha(S))} \subset E_{2}$. For any $\varepsilon>0$, take the finite-dimensional $G$-equivariant Schauder projection $p_{\varepsilon}: X \rightarrow X$ (see, e.g., [12, pages 69-70]) satisfying the property

$$
\left\|y-p_{\varepsilon}(y)\right\|<\varepsilon \quad(y \in X),
$$

and put $\alpha_{\varepsilon}:=p_{\varepsilon} \alpha$. Denote by $N\left(\alpha_{\varepsilon}\right)$ the solution set to the equation $\alpha_{\varepsilon}(y, u)=y,(y, u) \in S$. LEMMA 5.1. Under the above notations, ind $\left(N\left(\alpha_{\varepsilon}\right)\right) \leq \operatorname{ind}(N(\alpha))$ for all $\varepsilon$ small enough.

Proof. By continuity property of ind, there exists a closed invariant neighborhood $U$ $N(\alpha)$ such that ind $(U)=\operatorname{ind}(N(\alpha))$. Since $N(\alpha)$ is compact, without loss of generality, one can assume that $\mathcal{U}$ is a uniform $\delta$-neighborhood: $\mathcal{U}=\mathcal{U}_{\delta}(N(\alpha)):=\{z \in E \mid \| z-$ $N(\alpha) \|<\delta\}$ for $\delta>0$ small enough.

Let us show, first, that there exists $\varepsilon_{0}>0$ such that $N\left(\alpha_{\varepsilon}\right) \subset \bigcup_{\delta}(N(\alpha))$ for all $0<\varepsilon<\varepsilon_{0}$. Arguing indirectly, assume that for any $n \in \mathbb{N}$, there exists $\left(y_{n}, u_{n}\right) \in N\left(\alpha_{1 / n}\right)$ such that

$$
\left\|\left(y_{n}, u_{n}\right)-N(\alpha)\right\| \geq \delta .
$$

However, according to the definition of $X$ and inequality (5.8), one has $\alpha\left(y_{n}, u_{n}\right) \in X$ and $\left\|y_{n}-\alpha\left(y_{n}, u_{n}\right)\right\|<1 / n$. Since $X$ and the unit sphere of $U$ are compact, without loss of generality, one can assume that $y_{n} \rightarrow y_{*}$ and $u_{n} \rightarrow u_{*}$. Moreover, $\left(y_{*}, u_{*}\right) \in S$. By passing to the limit, one obtains $\alpha\left(y_{*}, u_{*}\right)=y_{*}$ that contradicts (5.9).

Therefore, the statement of Lemma 5.1 follows from monotonicity property of ind.

Return to the proof of Theorem 4.3 in the considered case. Take $\varepsilon$ small enough and the Schauder projection $p_{\varepsilon}$ satisfying (5.8). Let $\mathbb{R}^{k} \subset E_{2}$ be the invariant finite-dimensional subspace containing $p_{\varepsilon}(X)$. Put $\alpha_{\varepsilon}^{\prime}:=\left.\alpha_{\varepsilon}\right|_{\mathbb{R}^{k} \oplus U}$ and let $N\left(\alpha_{\varepsilon}^{\prime}\right)$ stand for the solution set to the equation $\alpha_{\varepsilon}^{\prime}(y, u)=y$. Combining the result obtained at the previous step with the monotonicity property of ind, one obtains

$$
\frac{p}{d} \leq \text { ind }\left(N\left(\alpha_{\varepsilon}^{\prime}\right)\right) \leq \text { ind }\left(N\left(\alpha_{\varepsilon}\right)\right) \leq \text { ind }(N(\alpha))
$$

Step 3 (infinite-dimensional kernel). Under the assumptions of Theorem 4.3, suppose that $p=\infty$ and take a finite-dimensional invariant subspace $V \subset U$ (cf. [20, Section 4 and Appendix C] or [21, page 57]). Put $E^{\prime}:=E_{2} \oplus V$ and $\alpha_{V}:=\left.\alpha\right|_{E^{\prime}}$. Denote by $N\left(\alpha_{V}\right)$ the solution set to the equation

$$
\alpha_{V}(y, u)=y \quad\left(y \in E_{2}, u \in V\right) .
$$


By monotonicity property, $N\left(\alpha_{V}\right) \subset N(\alpha)$ implies that ind $(N(\alpha)) \geq \operatorname{ind}\left(N\left(\alpha_{V}\right)\right)$. However (see Step 2), ind $\left(\alpha_{V}\right) \geq \operatorname{dim} V / d$. Bearing in mind that $\operatorname{dim} V$ can be chosen arbitrarily large (see again [20, Section 4 and Appendix C]), one obtains ind $(N(\alpha))=\infty$.

To complete the proof of Theorem 4.3, it remains to combine Steps 2 and 3 with Lemma 4.4 and the monotonicity property of ind.

Corollary 5.2. Under the assumptions of Theorem 4.3, suppose that $p=\infty$. Then $\operatorname{dim} N(a$, $f)=\infty$.

Proof. Arguing indirectly, assume that $\operatorname{dim} N(a, f)$ is finite. Then $N(a, f)$ is compact and, therefore, ind $(N(a, f))$ is finite as well. The obtained contradiction completes the proof.

\section{Application}

Let $\Lambda$ be a finite-dimensional linear space (thought of as a parameter space) and $b$ : $\mathbb{R}^{n} \times \Lambda \rightarrow \mathbb{R}^{n}$ a continuous map. Let, further, $C_{[0,2 \pi]}^{2 \pi}$ be the space of continuous functions $x:[0,2 \pi] \rightarrow \mathbb{R}^{n}$ with $x(0)=x(2 \pi)$ (equipped with the standard sup-norm). Put $E_{1}:=C_{[0,2 \pi]}^{2 \pi} \oplus \Lambda$ and $\|(x, \lambda)\|_{E_{1}}:=\|x\|+\|\lambda\|$.

Consider the following problem.

PRoвlem 6.1. Given a real number $R>0$, do there exist a differentiable $2 \pi$-periodic vectorfunction $x: \mathbb{R} \rightarrow \mathbb{R}^{n}$ and $\lambda \in \Lambda$ such that

$$
x^{\prime}(t)=b(x(t), \lambda)-\frac{1}{2 \pi} \int_{0}^{2 \pi} b(x(s), \lambda) d s \quad \forall t \in \mathbb{R},
$$

and $\|(x, \lambda)\|_{E_{1}}=R$ ? In addition, what can be said about the topological structure of the solution set $N(b)$ to the above problem?

Assume, in addition, $\Lambda$ is an (isometric) $S^{1}$-representation satisfying the condition

(*) $\Lambda^{S^{1}}=\{0\}$.

In particular, $\operatorname{dim} \Lambda$ is even and we will assume that

(**) $\operatorname{dim} \Lambda>0$.

Identify $C_{[0,2 \pi]}^{2 \pi}$ with the space of continuous functions $x: S^{1} \rightarrow \mathbb{R}^{n}$ and define on it the natural (isometric) $S^{1}$-representation: $(h x)(t)=x(t+\varphi)$, where $h=\exp (i \varphi) \in S^{1}$. Equip $E_{1}$ with the diagonal $S^{1}$-action.

Assume, further, the map $b$ from Problem 6.1 to be $S^{1}$-invariant in the second variable, that is,

$$
(* * *) b(x, h \lambda)=b(x, \lambda) \text { for all } x \in \mathbb{R}^{n}, \lambda \in \Lambda, h \in S^{1} .
$$

Proposition 6.2. Under the assumptions (*), (**), and (***), one has the following genus estimate for $N(b)$ :

$$
g(N(b)) \geq \frac{\operatorname{dim} \Lambda}{2}
$$

(in particular, $N(b) \neq \varnothing$ ). 
Proof. Observe, first, that by condition $(*)$,

$$
E_{1}^{S^{1}}=\{(x(\cdot), 0) \mid x(\cdot) \text { is a constant function }\} .
$$

Next, denote by $C_{[0.2 \pi]}$ the space of continuous functions from $[0,2 \pi]$ to $\mathbb{R}^{n}$ with the standard sup-norm, and let $d: D(d) \subset C_{[0,2 \pi]}^{2 \pi} \rightarrow C_{[0,2 \pi]}$ be the differentiation operator, where

$$
D(d)=\left\{x(\cdot) \in C_{[0,2 \pi]}^{[2 \pi]} \mid x(\cdot) \text { is smooth and } x^{\prime}(0)=x^{\prime}(2 \pi)\right\} .
$$

Obviously, $d$ is closed and $\operatorname{ker}(d)$ coincides with the set of constant functions.

Consider now the operator $a: D(a) \subset E_{1} \rightarrow C_{[0,2 \pi]}$ defined by

$$
a(x(\cdot), \lambda):=x^{\prime}(\cdot)
$$

Obviously, $D(a)=D(d) \oplus \Lambda$ and $\operatorname{ker}(a)=\operatorname{ker}(d) \oplus \Lambda$. Moreover, $a$ is still a closed operator. Put

$$
E_{2}:=\operatorname{Im}(a)=\left\{y(\cdot) \in C_{[0,2 \pi]} \mid \int_{0}^{2 \pi} y(s) d s=0, y(0)=y(2 \pi)\right\} .
$$

By direct computation, $E_{2}$ is a closed $S^{1}$-invariant subset of $C_{[0,2 \pi]}^{2 \pi}$, and $a$ is equivariant. Also, $E_{2}^{S^{1}}=\{0\}$.

Consider now a nonlinear continuous map $f$ determined by the right-hand side of (6.1):

$$
y(t):=f(x, \lambda)(t)=b(x(t), \lambda)-\frac{1}{2 \pi} \int_{0}^{2 \pi} b(x(s), \lambda) d s
$$

Obviously, $y(0)=y(2 \pi)$ and $\int_{0}^{2 \pi} y(s) d s=0$. Hence, $f$ takes $E_{1}$ to $E_{2}$. Moreover, since $\Lambda$ is assumed to be finite-dimensional, the map $f$ is $a$-compact. To check that $f$ is $S^{1}$ equivariant, take $h=\exp (i \varphi) \in S^{1}$. Using condition $(* * *)$, we have

$$
\begin{aligned}
f(h(x(t), \lambda)) & =f(h(x(t), h \lambda))=f(h(x(t), \lambda))-\frac{1}{2 \pi} \int g(h(x(s)), \lambda) d s \\
& =g(x(t+\varphi), \lambda)-\frac{1}{2 \pi} \int_{0}^{2 \pi} g(x(s+\varphi), \lambda) d s=h f(x(t), \lambda) .
\end{aligned}
$$

To complete the proof of Proposition 6.2, take the sphere $S_{R} \subset E_{1}$ and apply Theorem 4.3 (cf. condition $(* *)$ and Example 2.6(ii)).

Remark 6.3. (i) In Proposition 6.2, one can take any index theory (for $S^{1}$ ) satisfying the dimension property. Also, the segment $[0,2 \pi]$ is taken to simplify the presentation. 
(ii) In this paper, we restrict ourselves with the simplest illustrative example. In forthcoming papers, more involved applications (in particular, admitting closed operators with infinite-dimensional kernels) will be considered.

\section{Acknowledgments}

We are thankful to A. Kushkuley and H. Steinlein for improving our understanding of the subject. The first author acknowledges support from Grants IN-105803 from PAPIIT, Universidad Nacional Autónoma de México (UNAM) and C02-42563 from CONACYT (México). The second author acknowledges support from the Alexander von Humboldt Foundation. The third author acknowledges support from the Grant 01-05-00100 from RFBR.

\section{References}

[1] S. A. Antonyan, Retracts in categories of G-spaces, Izvestiya Akademii Nauk Armyanskoŭ SSR. Seriya Matematika 15 (1980), no. 5, 365-378, English translation in: Soviet Journal of Contemporary Mathematical Analysis 15 (1980), 30-43.

[2] J.-P. Aubin and I. Ekeland, Applied Nonlinear Analysis, John Wiley \& Sons, New York, 1984.

[3] T. Bartsch, Topological Methods for Variational Problems with Symmetries, Lecture Notes in Mathematics, vol. 1560, Springer, Berlin, 1993.

[4] V. Benci, On critical point theory for indefinite functionals in the presence of symmetries, Transactions of the American Mathematical Society 274 (1982), no. 2, 533-572.

[5] D. G. Bourgin, On some separation and mapping theorems, Commentarii Mathematici Helvetici 29 (1955), 199-214.

[6] G. Bredon, Introduction to Compact Transformation Groups, Academic Press, New York, 1972.

[7] Z. Dzedzej, Equivariant selections and approximations, Topological Methods in Nonlinear Analysis, Gdansk Scientific Society, Gdansk, 1997, pp. 25-31.

[8] B. D. Gel'man, The Borsuk-Ulam theorem in infinite-dimensional Banach spaces, Sbornik: Mathematics 193 (2002), no. 1, 83-91.

[9] __ An infinite-dimensional version of the Borsuk-Ulam theorem, Functional Analysis and Its Applications 38 (2004), no. 4, 1-5.

[10] P. Holm and E. H. Spanier, Involutions and Fredholm maps, Topology 10 (1971), no. 3, 203-218.

[11] J. Ize and A. Vignoli, Equivariant Degree Theory, De Gruyter Series in Nonlinear Analysis and Applications, vol. 8, Walter de Gruyter, Berlin, 2003.

[12] W. Krawcewicz and J. Wu, Theory of Degrees with Applications to Bifurcations and Differential Equations, John Wiley \& Sons, New York, 1997.

[13] A. Kushkuley and Z. I. Balanov, Geometric Methods in Degree Theory for Equivariant Maps, Lecture Notes in Mathematics, vol. 1632, Springer, Berlin, 1996.

[14] J. Mawhin and M. Willem, Critical Point Theory and Hamiltonian Systems, Applied Mathematical Sciences, vol. 74, Springer, New York, 1989.

[15] E. Michael, Continuous selections I, Annals of Mathematics 63 (1956), 361-382.

[16] W. Rudin, Functional Analysis, 2nd ed., McGraw-Hill, New York, 1991.

[17] H. Steinlein, Borsuk's antipodal theorem and its generalizations and applications: a survey, Topological Methods in Nonlinear Analysis (A. Granas, ed.), Sém Mathematics Sup., vol. 95, Presses de l' Université of Montréal, Montreal, 1985, pp. 166-235.

[18] I. A. Wolf, Spaces of Constant Curvature, McGraw-Hill, New York, 1967.

[19] C.-T. Yang, On theorems of Borsuk-Ulam, Kakutani-Yamabe-Yujobo and Dyson I, Annals of Mathematics 60 (1954), 262-282. 
[20] D. P. Zhelobenko, Introduction to Representation Theory, Factorial Press, Moscow, 2001.

[21] D. P. Zhelobenko and A. I. Shtern, Representations of Lie Groups, Nauka, Moscow, 1983.

Sergey A. Antonyan: Departamento de Matemáticas, Facultad de Ciencias, Universidad Nacional Autónoma de México, 04510 México DF, Mexico

E-mail address: antonyan@servidor.unam.mx

Zalman I. Balanov: Department of Mathematics and Computer Science, Netanya Academic College, 42365 Netanya, Israel

E-mail address: balanov@mail.netanya.ac.il

Boris D. Gel'man: Faculty of Mathematics, Voronezh State University, 1 Universitetskaya Pl., 394006 Voronezh, Russia

E-mail address: gelman_boris@mail.ru 


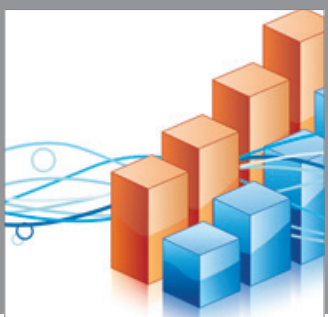

Advances in

Operations Research

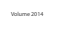

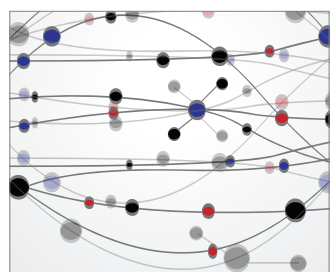

\section{The Scientific} World Journal
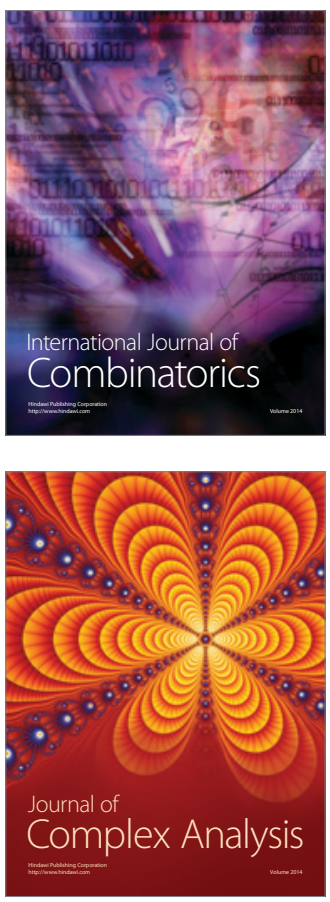

International Journal of

Mathematics and

Mathematical

Sciences
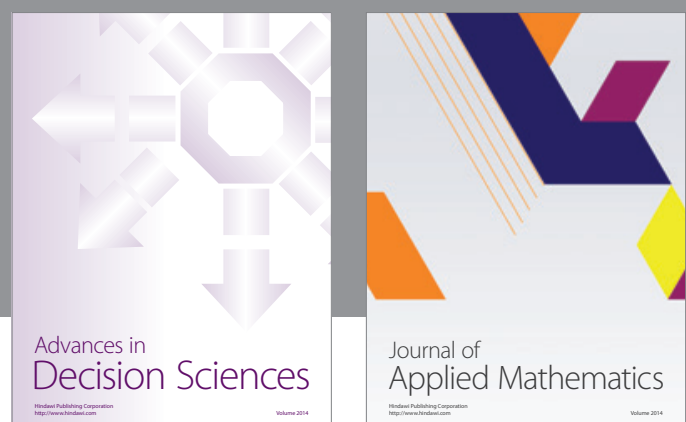

Journal of

Applied Mathematics
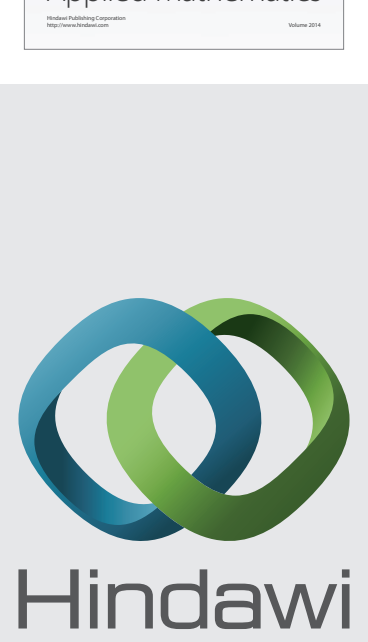

Submit your manuscripts at http://www.hindawi.com
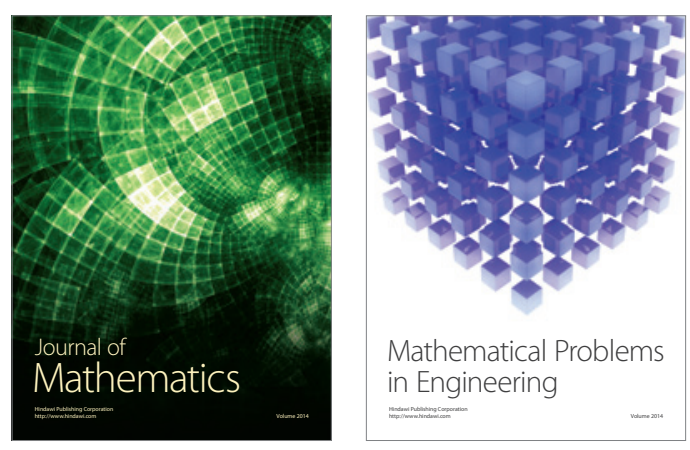

Mathematical Problems in Engineering
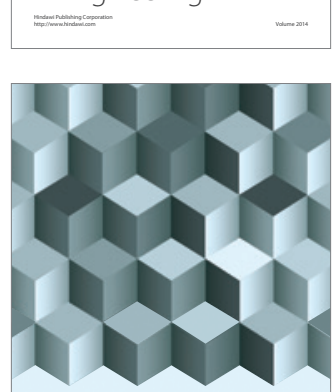

Journal of

Function Spaces
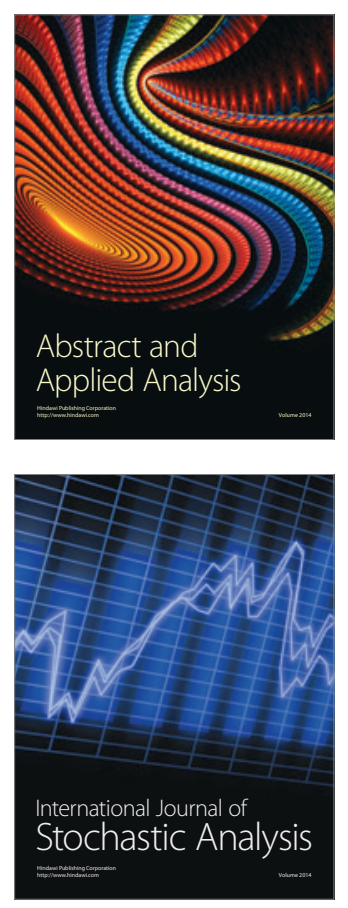

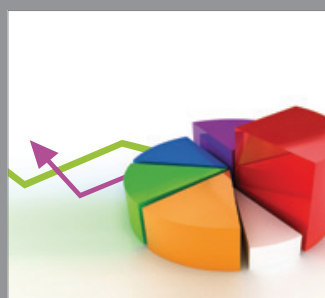

ournal of

Probability and Statistics

Promensencen
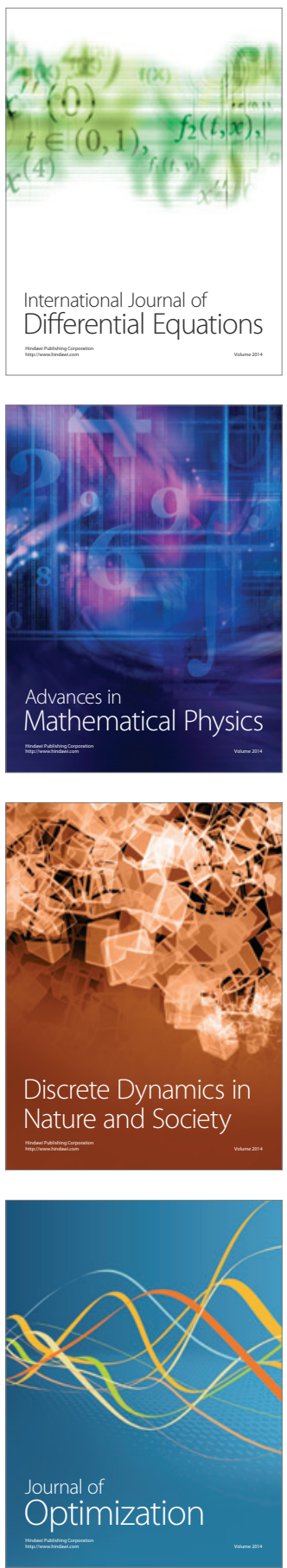